\title{
Benign Thyroid Diseases: Are There Gender-Specific Differences for Diagnosis and Treatment of Nontoxic Thyroid Nodules? Results from a 4-Year Retrospective Analysis of an Endocrine Tumor Board
}

\author{
Jasmin Mettler $^{a}$ Stella Armefti ${ }^{b}$ Matthias Schmidt ${ }^{a}$ Michael Faust ${ }^{c}$ \\ Marianne Engels ${ }^{d}$ Costanza Chiapponi ${ }^{b}$ \\ ${ }^{a}$ Department of Nuclear Medicine, Faculty of Medicine, University of Cologne and University Hospital Cologne, \\ Cologne, Germany; b Department of General, Visceral, Cancer and Transplantation Surgery, University of Cologne,

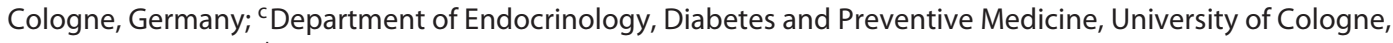 \\ Cologne, Germany; ${ }^{\mathrm{d}}$ Department of Pathology, University of Cologne, Cologne, Germany
}

\author{
Keywords \\ Gender · Benign thyroid disease $\cdot$ Bethesda $\cdot$ Fine needle \\ biopsy
}

\begin{abstract}
Introduction: Benign nodular goiters are endemic in Germany and diagnostic thyroidectomy is one of the most frequent surgical procedures. Less than $10 \%$ result in a malignant finding which is a poor ratio in comparison to other European countries. There is a female preponderance in almost all thyroid pathologies according to the current literature but not much is known concerning gender-specific differences in thyroid nodules. Objective: We aimed at finding gender-specific differences in diagnosis and treatment of nontoxic thyroid nodules and focused on preoperative workup as well as cytological and histological findings of patients with solitary and multinodular nontoxic goiters. Methods: We retrospectively analyzed 392 cases out of 693 thyroid cases managed interdisciplinarily by the endocrine board of a university center between January 2015 and December 2018 (4 years). Results: The combination of fine needle biopsy and interdisciplinary case discussion resulted in a rate of malignancy of $28.9 \%$ in patients undergoing surgery for solitary and multinodular nontoxic goiter. Although there was no significant
\end{abstract}

gender-specific difference in the distributions and malignancy rates of Bethesda categories, male patients had a significantly higher malignancy rate of $40 \%$ in our collective ( $p=$ 0.04). Conclusions: Surgical treatment for male patients with suspicious hypofunctioning thyroid nodules should be favored. However, well-designed prospective studies are required to investigate gender-specific recommendations for the treatment of benign thyroid diseases in the future.

(c) 2020 S. Karger AG, Basel

\section{Introduction}

Although euthyroid nodular goiter is very common and more frequent in women, there are not much data concerning gender-specific features of female and male nodules. A recent study showed a greater likelihood of cytologically verified presence of intranodular chronic lymphocytic thyroiditis in females and suggested that the presence/absence of intranodular chronic lymphocytic thyroiditis might be associated with some sexually dimorphic characteristics of thyroid nodules [1].

J.M. and S.A. contributed equally to this work. 
Table 1. Distribution of thyroid disorders in male and female patients whose cases were discussed in the endocrine board within 4 years

\begin{tabular}{lccc}
\hline Thyroid disorders & $\begin{array}{l}\text { All } \\
(n=693)\end{array}$ & $\begin{array}{l}\text { Male } \\
(n=209,30.2 \%)\end{array}$ & $\begin{array}{l}\text { Female } \\
(n=484,69.8 \%)\end{array}$ \\
\hline Nontoxic solitary nodule & $194(27.9)$ & $57(29.4)$ & $137(70.6)$ \\
Nontoxic multinodular goiter & $198(28.6)$ & $52(26.3)$ & $146(73.7)$ \\
Toxic solitary and multinodular goiter & $40(5.8)$ & $12(30)$ & $28(70)$ \\
AITD & $71(10.2)$ & $20(28.2)$ & $51(71.8)$ \\
All thyroid carcinomas & $178(25.7)$ & $63(35.4)$ & $115(64.6)$ \\
Other & $12(1.7)$ & $5(41.6)$ & $7(58.3)$ \\
\hline
\end{tabular}

Data are presented as $n(\%)$. A total of 392 patients were presented with the primary diagnosis of solitary ( $n=194)$ or multinodular $(n=198)$ nontoxic goiter. This group made up $56.5 \%$ of all thyroid cases discussed. AITD, autoimmune thyroid disease.

Gender-specific differences of female and male nodules might play a role in defining therapeutic pathways for solitary nodules and multinodular goiter. In fact most of the cases undergoing "diagnostic surgery" turn out to be benign: in Germany 14 out of 15 procedures (as opposed to 6:7 in Italy and 4:5 in England and Scandinavian countries [2]). In a recent German analysis of 12,888 patients with benign thyroid pathologies, $68 \%$ of cases were operated on to rule out malignancy [3]. However, preoperative tools like measurement of serum calcitonin and fine needle biopsy (FNB) had been performed in only 62 and $12 \%$ of cases, respectively [3].

At our university institution all patients with thyroid disease requiring "diagnostic surgery" are discussed in the endocrine tumor board, which takes place weekly, in order to ensure board certification ("Deutsche Krebsgesellschaft," DKG). It also offers patients an adequate preoperative diagnostic workup, avoiding overtreatment. With a female preponderance in all thyroid pathologies, in this retrospective study, we analyzed whether systematic gender-specific differences are to be found in the results of the diagnostic workup, in the recommendations of our tumor board, and in the histological findings of patients with solitary and multinodular nontoxic goiter.

\section{Materials and Methods}

The present study retrospectively evaluated the applications and recommendations of 693 patients with thyroid diseases discussed in our endocrine board between January 1, 2015, and December 31,2018 . All recommendations were given by the same interdisciplinary team including endocrinology, nuclear medicine, and endocrine surgery. All cases which were primarily presented as malignant were excluded. We focused on gender-specific correlations concerning the diagnostic results, the board's recommendation of surgical treatment, and the final histopathologic result of patients with hypofunctioning thyroid nodules (HTN) or thyroid nodules with suspicious ultrasound features $(n=392)$. Because this study is based on retrospectively analyzed data of pa-
Table 2. Distribution of Bethesda categories in male and female patients.

\begin{tabular}{llcl}
\hline Bethesda & $\begin{array}{l}\text { Total } \\
(n=213)\end{array}$ & $\begin{array}{l}\text { Male } \\
(n=57)\end{array}$ & $\begin{array}{l}\text { Female } \\
(n=156)\end{array}$ \\
\hline I & $31(14.5)$ & $7(12.3)$ & $24(15.4)$ \\
II & $47(22.1)$ & $15(26.3)$ & $32(20.5)$ \\
III & $72(33.8)$ & $16(28.1)$ & $56(35.9)$ \\
IV & $28(13.2)$ & $5(8.8)$ & $23(14.7)$ \\
V & $23(10.8)$ & $8(14.0)$ & $15(9.6)$ \\
VI & $12(5.6)$ & $6(10.5)$ & $6(3.9)$ \\
\hline
\end{tabular}

Data are presented as $n(\%)$. There were no gender-specific differences.

tients who underwent common treatment at the university hospital, a specific consent from individual participants was not required.

FNB of thyroid nodules was performed in patients with HTN or when nodules had ultrasound characteristics that were suspicious for malignancy. More than $90 \%$ of the FNB in this study were performed in the policlinic of the nuclear medicine department. Biopsies were guided ultrasonographically in an out-of-plane technique. For sampling 25 -gauge $(0.5 \times 25 \mathrm{~mm})$ needles were used. The cannulas were applied to $20-\mathrm{mL}$ plastic syringes which were fixed in syringe holders for single-handed operability. Samples were either applied on specimen slides or injected into SurePath ${ }^{\mathrm{TM}}$ vials for liquid-based cytology where specimens are flushed out of the needle with the preservative fluid. Liquid-based cytology samples went through a cell enrichment process and were automatically prepared on an approximate monolayer. Hematoxylin and eosin stain and May-Gruenwald-Giemsa stain were used on the air-dried specimens. Papanicolaou stain was used on liquid-based cytology specimens. Cytological findings were transferred into the Bethesda scoring system. Nodules were classified according to the 6-category Bethesda system.

Surgeries were performed as hemithyroidectomies and thyroidectomies by two experienced and certified Fellows of the European Board of Surgery (FEBS) for endocrine surgery. The surgical unit performs about 200 cervical endocrine procedures per year. For all procedures magnifying glasses and neuromonitoring were used. All nodules were analyzed intraoperatively as frozen 
Table 3. Percentage and age of male and female patients receiving surgical treatment in each Bethesda group

\begin{tabular}{|c|c|c|c|c|c|c|}
\hline \multirow[t]{2}{*}{ Total } & \multicolumn{3}{|c|}{ Patients undergoing surgery } & \multicolumn{3}{|l|}{ Age, years } \\
\hline & male & female & $p$ & male & female & $p$ \\
\hline Total & $35 / 57(61.4)$ & $114 / 156(73.1)$ & 0.13 & $56.7 \pm 13.9$ & $52.3 \pm 16.3$ & 0.05 \\
\hline Bethesda I & $4 / 7(57.1)$ & $19 / 24(79.2)$ & 0.35 & $54.0 \pm 15.7$ & $53.3 \pm 15.1$ & 0.92 \\
\hline Bethesda II & $4 / 15(26.7)$ & $19 / 32(59.4)$ & $0.03^{*}$ & $57.4 \pm 11.2$ & $48.5 \pm 16.5$ & $0.036^{*}$ \\
\hline Bethesda III & $11 / 16(68.8)$ & $43 / 56(76.8)$ & 0.81 & $56.5 \pm 16.5$ & $54.9 \pm 16$ & 0.72 \\
\hline Bethesda IV & $3 / 5(60)$ & $15 / 23(65.2)$ & 0.54 & $54.4 \pm 15$ & $54.5 \pm 18.7$ & 0.99 \\
\hline Bethesda V & $8 / 8(100)$ & $14 / 15(93.3)$ & 0.46 & $59.8 \pm 11.3$ & $47.9 \pm 12.7$ & $0.038^{*}$ \\
\hline Bethesda VI & $5 / 6(83.3)$ & $4 / 6(66.7)$ & 0.57 & $56.7 \pm 17.3$ & $46 \pm 21.3$ & 0.36 \\
\hline
\end{tabular}

Data are presented as $n(\%)$ or mean \pm SD as appropriate. In the Bethesda II group the rate of women undergoing surgery was significantly higher than that of men $(59.4$ vs. $26.7 \%, p=0.03)$. These women were almost 10 years younger than the men in the same group $(p=0.036)$. The difference was similar in the Bethesda $\mathrm{V}$ group ( 47.9 vs. $59.8 \%, p=0.038$ ), although the total collective of men undergoing surgery was slightly older than the female collective (56.7 vs. 52.3 years, $p=0.05)$. ${ }^{*}$ Statistically significant difference.

section samples. There were no postoperative bleedings and the recurrent laryngeal nerve palsy rate tested by the Ear-NoseThroat Department on the second postoperative day was approximately $2 \%$.

Data were analyzed using IBM SPSS Statistics for Windows, Version 25.0 (IBM, Armonk, NY, USA). Continuous variables were compared using the two-tailed Student $t$ test, and categorical variables were compared using the $\chi^{2}$ test or Fisher's exact test.

\section{Results}

In the 4-year time period, 842 patients were discussed in the interdisciplinary board. Of these, 693 patients $(82.3 \%)$ suffered from thyroid disorders.

Table 1 shows the frequency distribution of all thyroid disorders discussed; 392 patients were presented with the primary diagnosis of solitary $(n=194)$ or multinodular ( $n=198)$ nontoxic goiter ( $56.5 \%$ of all thyroid cases). The mean age of these patients was $54.9 \pm 16.5$ years; $72.2 \%$ of them were female.

In the 392 cases of solitary or multinodular nontoxic goiter, ultrasound and laboratory examinations including calcitonin were performed in all cases $(100 \%)$ as a requirement for presentation to the board.

In total, 356 of these patients had a scintigraphic hypofunctional (HTN) or indifferent nodule $(90.8 \%) ; 68.9 \%$ of them were female and they were significantly younger (53.0 \pm 16 vs. $57.2 \pm 14$ years) than the male patients ( $p=$ $0.02)$.

FNB was performed in 213 patients with sonographic and scintigraphic suspicious (hypofunctional or indifferent) nodules (59.8\%). The rate of FNB in the female group was significantly higher ( 64.5 vs. $50 \%, p=0.014)$. There was no gender-specific difference in age in this diagnostic group ( $52.2 \pm 16$ vs. $56.7 \pm 13.8$ in male patients, $p=0.07$ ).
There was also no gender- or age-specific difference in the distribution of the cytological categories diagnosed (Table 2).

Surgery was performed in 203 of the 392 patients with solitary or multinodular nontoxic goiter (51.8\%). Among all patients receiving surgical treatment, male patients were significantly older than female patients $(56.7 \pm 13.9$ vs. $52.3 \pm 16.3, p=0.05)$. However, comparing males and females separately, there were no significant age differences between patients undergoing surgery and those not ( $p=0.8$ and 0.09 , respectively). FNB had been performed in $73.4 \%$ of all operated patients, and of all patients who had undergone FNB, $69.9 \%$ were treated surgically.

There was a significant higher percentage of women undergoing diagnostic surgery in the group of patients with FNB results that had been diagnosed as low-risk for thyroid cancer (Bethesda II, $p=0.03$ ). In all other Bethesda categories, there was no significant gender-specific difference concerning the choice of surgical treatment (Table 3). Female patients receiving a Bethesda II and V diagnosis and undergoing surgery were significantly younger than their male counterparts $(48.5 \pm 16.5$ vs. $57.4 \pm$ $11.2, p=0.036$ and $47.9 \pm 12.7$ vs. $59.8 \pm 11.3, p=0.038$, respectively).

A final histological diagnosis of malignancy was found in $26.6 \%$ of all patients with solitary or multinodular nontoxic goiter undergoing surgery $(n=54 / 203)$ and in $28.9 \%$ of those who had received FNB and subsequent surgery (49/149). There were no age-specific differences. Male patients who underwent surgery after FNB had a significantly greater likelihood of malignant thyroid pathologies $(n=14 / 35,40 \%)$ than women $(p=0.04)$. However, there was no further significant gender-specific difference among single Bethesda categories concerning malignancy. 
Table 4. Rates of malignancy in each Bethesda category

\begin{tabular}{lcccc}
\hline & $\begin{array}{l}\text { Literature } \\
{[4,5]}\end{array}$ & $\begin{array}{l}\text { Total } \\
(n=149)\end{array}$ & $\begin{array}{l}\text { Male } \\
(n=35)\end{array}$ & $\begin{array}{l}\text { Female } \\
(n=114)\end{array}$ \\
\hline I & $5-10$ & 17.4 & 25 & 15.8 \\
II & $0-3$ & 8.7 & 25 & 5.3 \\
III & $10-30$ & 14.8 & 9.1 & 16.3 \\
IV & $25-40$ & 22.2 & 0 & 26.7 \\
V & $50-75$ & 77.3 & 75 & 78.6 \\
VI & $97-99$ & 88.9 & 100 & 75 \\
\hline
\end{tabular}

Data are presented as \%. This table depicts the values commonly found in the literature (first column), those found in our collective ( $n=149$; second column) who underwent FNB and subsequent thyroid surgery for Bethesda I, II, III, IV, V, and VI ( $n=$ $23,23,54,18,22$, and 9 , respectively). Columns 3 and 4 present the rates for male and female patients, respectively. The differences between men and women are not significant.

Malignancy rates for Bethesda categories III and V roughly accord to the reference rates found in the literature $[4,5]$ (Table 4). Malignancy rates for Bethesda I and II exceeded the referential malignancy rates for both male and female patients. In particular, there were 2 cases of malignancy despite a Bethesda II diagnosis: surgery revealed a $\mathrm{pT} 1 \mathrm{~b}$ papillary thyroid cancer in a male patient with a highly suspicious nodule in ultrasound (1/4). In the second case surgery was performed in a female patient despite a Bethesda II diagnosis, after biopsy of her osteolytic lesions had revealed metastases of a papillary thyroid cancer $(1 / 19)$. The primary tumor could not be found in the surgical specimen (right lobectomy) and might have been misdiagnosed in the left lobectomy specimen removed several years before elsewhere.

No male patient within the Bethesda IV category had a malignant histopathological finding. Female patients within the Bethesda VI category had a lower rate of malignant histopathological findings, as expected.

\section{Discussion}

The analysis of 842 cases from 4 years of the interdisciplinary endocrine board showed that a major fraction consisted of thyroid cases $(693,82.3 \%)$. Solitary and multinodular nontoxic goiter as a primary diagnosis was the reason for presentation in more than half of the cases (56.5\%), showing the relevance of this disease for the endocrine board. There was a female preponderance in all thyroid pathologies presented. In the group of solitary and multinodular nontoxic goiter, women represented $72.2 \%$ of all cases. For this reason, we decided to search for gender-specific differences in diagnosis and treatment in this collective.
As a first result, we found that a high percentage of patients with solitary and multinodular nontoxic goiter received FNB at our institution (69.6\% of patients with sonographic and scintigraphic suspicious nodules). This rate is higher than that described recently for Germany [3]. This rate was significantly higher among female patients than males $(p=0.014)$. This might indicate a greater willingness of women to undergo diagnostic procedures. FNB represents a central tool for diagnostic workup. The cytopathological result justifies either a conservative or nonoperative (interventional, ablative) treatment of HTN (Bethesda II) or guides for surgery in patients receiving a Bethesda IV, V, or VI diagnosis. The rates of malignancy for the Bethesda III group vary among institutions and general recommendations include repeat FNP, molecular testing, or "diagnostic surgery." These 3 options are usually offered to patients. In contrast, surgery is recommended for patients with Bethesda IV, V, and VI, since the risk of malignancy is high.

There was no gender-specific difference in the distribution of the Bethesda categories in this study and the rate of high-risk FNB categories (Bethesda IV-VI) was not significantly higher in females, confirming results of Arena and Benvenga [1] and Todorovic et al. [6].

Surgery was performed in almost half of the cases of solitary and multinodular nontoxic goiter discussed in the endocrine board (51.8\%). Among patients undergoing surgical treatment, male patients were significantly older than females and this might be a possible explanation for the significantly higher rate of malignancy in males receiving FNB and surgery $(40 \%, p=0.04)$. A similar result was described by Todorovic et al. [6]. Symptoms or cosmetic reasons for surgery in female patients are a further possible explanation for the higher rate of malignancy in males; for example, the patients choosing surgery despite a Bethesda II diagnosis were significantly younger $(p=$ 0.036). However, our data might also suggest that male patients with HTN and sonographic features suspicious for malignancy should be treated operatively.

The general high rate of malignancy in all patients undergoing FNB and surgery for solitary or multinodular nontoxic goiter (1:3.4) emphasizes the quality of the preoperative diagnostic workup at our center, reducing the amount of unnecessary procedures. There were of course cases in which FNB was not performed: sometimes because of anticoagulatory medication, in some cases because patients refused it, and in some other cases because the patients had already decided that they wanted to undergo surgery. There was no gender- or age-specific difference in this group of patients with upfront surgery without FNP.

In this study, malignancy rates for Bethesda categories III and V roughly accord to the reference rates found in the literature $[4,5]$. Malignancy rates for Bethesda I and 
II exceeded the referential malignancy rates for both male and female patients. This discrepancy can be partly explained by patient preselection. Even though FNB was neither diagnostic nor suspicious, other reasons like suspicious ultrasound characteristics or clinical presentation of nodules still led the physician to advocate surgery. Thus, surgery was appropriately recommended and performed in 1 of 4 male patients receiving surgical treatment despite a Bethesda II diagnosis, revealing a pT1b papillary thyroid cancer. The small number of patients accounts for the surprisingly high rate of malignancy of $25 \%$ recorded in this group. In the other case of malignancy despite a Bethesda II FNB result, a female patient, who had received left hemithyroidectomy several years before with a benign histological diagnosis, presented with osteolytic lesions, which biopsy revealed to be metastases of a papillary thyroid cancer. Although FNP of the right thyroid lobe was classified as Bethesda II, right hemithyroidectomy was performed. No primary tumor could be found in the right thyroid lobe though. The patient subsequently underwent radioiodine treatment.

No male patient within the Bethesda IV category had a malignant histopathological finding. This result might be due to the history of iodine deficiency in Germany: most Bethesda IV nodules turned out to be follicular adenomas. The probability of follicular carcinoma in Bethesda IV findings was lower than in other countries like the USA. Thus, FNB cannot differentiate between adenoma and follicular carcinoma by cytopathology.

The low malignancy rate in female patients with Bethesda VI findings can be explained by the very low number of follicular carcinomas in our patient cohort $(n=9)$.

Limitations of this study in general are due to the retrospective and unicentric nature of the analysis, and the small patient collective analyzed. All cytological and pathologic diagnoses were taken in a single pathologic center and all recommendations were taken in an interdisciplinary consent, avoiding the bias which might be found in an exclusively surgical collective. Surgical straightforward cases, which were not discussed in our tumor board, have not been included in the present study. In addition, hyperthyroidism (including thyroiditis and autoimmune disease) was not included in this analysis, as it accounts for approximately only $10 \%$ of indications.

To our knowledge the current treatment of all thyroid disease includes no gender-specific differences except for Graves disease: the ATA Guidelines 2016 [7] (but not the ETA Guidelines [8]) suggest a gender-specific progression, and report of "lower remission rates in men, smokers (especially men) and those with large goiters $(>80$ $\mathrm{mL}$ )", thus suggesting that male patients might be more suitable candidates for a definitive treatment. This statement is based only on a few studies showing a worse outcome of medical treatment of Graves disease in men [9-
12]. One reason for this gender-specific difference might be that men often have a larger goiter size than women, which seems to be associated with severe immune and biochemical disorders in the case of Graves disease [12, 13]. Whether male gender is a negative prognostic factor for Graves disease is still a very controversial issue with only few retrospective studies reporting inconsistent results [14-16]. This inconsistency is often due to the different definition used for relapse (in terms of months after the discontinuation of treatment) and/or remission (in terms of time of diagnosis). Diker-Cohen et al. [17], who used the ATA definitions, found no significant differences in the remission and relapse rates of male and female patients. They even reported less severe adverse effects of antithyroid treatment and less frequent discontinuation of treatment in men [17].

For solitary and multinodular nontoxic goiters, which account for roughly half of all cases discussed in our endocrine tumor board, the combination of ultrasound and FNP reduces the amount of "unnecessary surgeries," increasing the rate of malignancy to approximately 1:3.4 of all cases. However, there were no significant gender-specific differences which might influence the current management of benign thyroid disease in our patients.

\section{Conclusion}

This retrospective study focused on gender-specific variations in diagnosis and treatment of euthyroid patients with HTN. We found no significant difference in preoperative workup but a significantly higher malignancy rate in male than in female patients. Comparing FNB results sorted into distinct Bethesda categories I-V, there was no gender-specific difference in these distinct categories. Thus, indication for surgery should still be guided by FNB and Bethesda categories but generally more liberally in men than in women, which is routinely performed in most centers already.

\section{Acknowledgments}

We thank our colleagues who participate in the endocrine board on a regular basis. We also thank the referring physicians in our region for entrusting their patients to our care.

\section{Statement of Ethics}

Given the retrospective nature of the research presented in this manuscript no ethical committee was involved. All patients agreed that their data be used anonymously for research purposes, by being treated at a university hospital. This paper will not be simultaneously submitted to any other publication during the review process. 


\section{Disclosure Statement}

The authors declare to have no conflicts of interest and no nonfinancial relationships that may have influenced the writing of the manuscript.

\section{Funding Sources}

There was no financial funding for this study.

\section{Author Contributions}

Each author made substantial contributions to the conception of the work. J.M. is responsible for the acquisition, S.A. for the analysis, and J.M., S.A., and C.C. for the interpretation of data. All authors participated in drafting the work. M.S., M.E., and M.F. revised it critically for important intellectual content; all authors approved the final version to be published and agreed to be accountable for all aspects of the work in ensuring that questions related to the accuracy or integrity of any part of the work are appropriately investigated and resolved.

\section{References}

1 Arena S, Benvenga S. Gender-specific correlation of intranodular chronic lymphocytic thyroiditis with thyroid nodule size, echogenicity, and histologically-verified cytological class of malignancy risk. J Clin Transl Endocrinol. 2018 Oct;14(December):39-45.

2 Bartsch DK, Luster M, Buhr HJ, Lorenz D, Germer CT, Goretzki PE; German Society for General and Visceral Surgery. Indications for the Surgical Management of Benign Goiter in Adults. Dtsch Arztebl Int. 2018 Jan 8;115(102):1-7.

3 Bartsch DK, Dotzenrath C, Vorländer C, Zielke A, Weber T, Buhr HJ, et al. The StuDoQ/ Thyroid Study Group TSS. Current Practice of Surgery for Benign Goitre-An Analysis of the Prospective DGAV StuDoQ|Thyroid Registry. J Clin Med. 2019 Apr;8(4):E477.

4 Nga MN. Updates in thyroid cytology. In: Parameswaran R, Agarwasl A, editors. Evidence-based endocrine surgery. Singapore: Springer; 2018.

5 Cibas ES, Ali SZ. The 2017 Bethesda System for Reporting Thyroid Cytopathology. Thyroid. 2017 Nov;27(11):1341-6.

6 Todorovic E, Sheffield BS, Kalloger S, Walker $\mathrm{B}$, Wiseman SM. Increased Cancer Risk in Younger Patients with Thyroid Nodules Diagnosed as Atypia of Undetermined Significance. Cureus. 2018 Mar;10(3):e2348.
7 Carlé A, Pedersen IB, Knudsen N, Perrild H, Ovesen L, Laurberg P. Gender differences in symptoms of hypothyroidism: a populationbased DanThyr study. Clin Endocrinol (Oxf). 2015 Nov;83(5):717-25.

8 Vadiveloo T, Donnan PT, Murphy MJ, Leese GP. Age- and gender-specific TSH reference intervals in people with no obvious thyroid disease in Tayside, Scotland: the Thyroid Epidemiology, Audit, and Research Study (TEARS). J Clin Endocrinol Metab. 2013 Mar; 98(3):1147-53.

9 Park SJ, Kim HI, Oh HK, Kim TH, Jang HW, Chung JH, et al. Age- and gender-specific reference intervals of TSH and free T4 in an iodine-replete area: Data from Korean National Health and Nutrition Examination Survey IV (2013-2015). PLoS One. 2018 Feb; 13(2):e0190738.

10 Magri F, Zerbini F, Gaiti M, Capelli V, Ragni A, Rotondi M, et al. Gender influences the clinical presentation and long-term outcome of Graves disease. Endocr Pract. 2016 Nov; 22(11):1336-42.

11 Ross DS, Burch HB, Cooper DS, Greenlee MC, Laurberg P, Maia AL, et al. 2016 American Thyroid Association Guidelines for Diagnosis and Management of Hyperthyroidism and Other Causes of Thyrotoxicosis. Thyroid. 2016 Oct;26(10):1343-421.
12 Kahaly GJ, Bartalena L, Hegedüs L, Leenhardt L, Poppe K, Pearce SH. 2018 European Thyroid Association Guideline for the Management of Graves' Hyperthyroidism. Eur Thyroid J. 2018 Aug;7(4):167-86.

13 Allahabadia A, Daykin J, Holder RL, Sheppard MC, Gough SC, Franklyn JA. Age and gender predict the outcome of treatment for Graves' hyperthyroidism. J Clin Endocrinol Metab. 2000 Mar;85(3):1038-42.

14 Kim KW, Park YJ, Kim TY, Park DJ, Park KS, Cho BY. Susceptible alleles of the CD40 and CTLA-4 genes are not associated with the relapse after antithyroid withdrawal in Graves' disease. Thyroid. 2007 Dec;17(12):1229-34.

15 Anagnostis P, Adamidou F, Polyzos SA, Katergari S, Karathanasi E, Zouli C, et al. Predictors of long-term remission in patients with Graves' disease: a single center experience. Endocrine. 2013 Oct;44(2):448-53.

16 Magri F, Zerbini F, Gaiti M, Capelli V, Ragni $\mathrm{A}$, Rotondi $\mathrm{M}$, et al. Gender influences the clinical presentation and long-term outcome of Graves disease. Endocr Pract. 2016 Nov; 22(11):1336-42.

17 Diker-Cohen T, Duskin-Bitan H, Shimon I, Hirsch D, Akirov A, Tsvetov G, et al. Disease presentation and remission rate in Graves disease treated with antithyroid drugs: is gender really a factor? Endocr Pract. 2019 Jan;25(1): 43-50. 